\title{
LA DIRECCIÓN Y LA DISTANCIA EN EL ESTABLECIMIENTO DE LA COHERENCIA REFERENCIAL DURANTE EL PROCESAMIENTO DE TEXTOS ACADÉMICOS ESCRITOS EN ESPAÑOL
}

\author{
THE EFFECT OF DIRECTION AND DISTANCE IN THE \\ ESTABLISHMENT OF REFERENTIAL COHERENCE DURING \\ ACADEMIC TEXT WRITTEN IN SPANISH PROCESSING
}

\author{
ROMUALDO IBÁÑEZ O ${ }^{1}$ \\ Pontificia Universidad Católica de Valparaíso \\ Romualdo.ibanez@ucv.cl \\ ANDREA SANTANA C. \\ Utrecht University, Países Bajos \\ A.C.SantanaCovarrublas@uu.nl \\ FELIPE CORNEJO T. \\ Facultad de Psicología, Universidad San Sebastián \\ felipe.cornejo@uss.cl
}

\section{RESUMEN}

Durante los últimos cuarenta años se ha desarrollado un gran número de trabajos respecto de la coherencia referencial y de su procesamiento. La mayoría de estos estudios asume la desambiguación de pronombres como un fenómeno invariable. Desde nuestra perspectiva, debido al tipo y cantidad de conocimiento involucrado durante la lectura de textos académicos, el modo en que ciertos factores afectan el establecimiento de la coherencia referencial es diferente de cómo lo hacen durante la lectura de textos no especializados. En este trabajo se estudia el efecto de la dirección y de la distancia referencial en el establecimiento de la coherencia referencial durante el procesamiento de textos académicos escritos en español. Para ello se llevaron a cabo dos experimentos en los que se midieron los tiempos y la precisión de respuesta en el procesamiento de segmentos discursivos correspondientes a textos de Psicología y de Ingeniería en Construcción. Al

\footnotetext{
${ }^{1}$ Autor principal: Romualdo Ibáñez O. Tel.: +56 322274408 Correo electrónico: romualdo.ibanez@ucv.cl. La investigación aquí presentada se realizó en el marco del proyecto Fondecyt 1120519 .
} 
igual que en estudios de procesamiento del discurso general, los resultados demuestran que durante el procesamiento de textos académicos escritos en español tanto la variable distancia como la variable dirección tienen un efecto significativo en el establecimiento de la coherencia referencial. No obstante, en este caso, la variable dirección se comporta de manera diferente, pues el procesamiento es más eficiente en la condición catafórica.

Palabras Clave: Coherencia referencial, discurso académico, distancia referencial, dirección referencial.

\section{ABSTRACT}

For the last forty years, a great number of studies regarding referential coherence and its processing has been carried out. In most of them, pronoun disambiguation is understood as an invariable phenomenon. From our perspective, due to the type and amount of knowledge involved, during academic reading, the way certain factors affect this type of processing is different from the way they do during non-specialized texts reading. In this work, we study the effect of referential distance and referential direction on referential coherence processing when reading academic texts written in Spanish. To do so, we carried out two experiments in which we assessed time and accuracy of answers in the processing of discourse segments corresponding to texts from Psychology and Construction Engineering. Equal to the case of studies on general discourse processing, results show that when reading academic texts written in Spanish, referential direction and referential distance have a significant effect in the establishment of referential coherence. But, in the case of academic discourse processing, processing is more efficient in cataphoric condition.

Keywords: Referential coherence, academic discourse, referential distance, referential direction.

Recibido: 05.01.2015. Aceptado: 03.08.2015.

\section{INTRODUCCIÓN}

- $n$ la actualidad existe una gran cantidad de estudios acerca del procesamienCto de la coherencia referencial, los que además han sido desarrollados desde diversas perspectivas teórico-metodológicas. Tal cantidad y diversidad de estudios queda de manifiesto en el trabajo compilatorio de Schwarz-Friesel, Consten y Knees (2007), así como también en el de Sanders y Gernsbacher (2004). En estas revisiones también se evidencia que ciertos rasgos del fenómeno han provocado constante interés en los investigadores. Entre estos rasgos se encuentra la dirección referencial, la que ha dado origen a un gran número de trabajos sobre el procesamiento anafórico (Garnham, 2001) y no tantos acerca del procesamiento catafóri- 
co (Gernsbacher, 1990). Otro rasgo que ha llamado la atención de los especialistas es la distancia referencial, considerada como fundamental en las teorías de la accesibilidad (Ariel, 2001; Givón, 1983, 1984) y estudiada como una variable en el procesamiento de la coherencia referencial (Clark y Sengul, 1979).

Sin embargo, la gran mayoría de las investigaciones aborda este proceso psicolingüístico como un fenómeno independiente del contexto y, en este sentido, lo asume como invariable. Tal situación es destacada por Garnham, Garrod y Sanford (2006), quienes sostienen que los materiales utilizados en gran parte de las investigaciones psicolingüísticas son descontextualizados y poco cercanos a lo que sucede en la vida real. De igual modo, Sanders (1997) destaca la necesidad de hacer explícitas las nociones teóricas en torno al discurso en textos naturales y no solo a través de ejemplos construidos para llevar a cabo experimentos. Ariel (2007), por su parte, plantea que a pesar de que la variación lingüística es algo que no está en discusión, la teoría de la accesibilidad, como una manera de describir la coherencia referencial, solo da cuenta del funcionamiento de las expresiones referenciales en términos generales, sin considerar las diferencias impuestas por los contextos de uso. En este punto, cabe considerar que los textos, como manifestaciones concretas de una lengua, poseen características impuestas tanto por la función comunicativa que cumplen (Halliday, 1994), como por el contexto en el que circulan (Bhatia, 2004; Hyland, 2004; Biber, Connor y Upton, 2007), lo que hace posible no solo hablar de géneros discursivos (Swales, 1990), sino que también de tipos de discurso, tales como el discurso académico, el discurso científico o el discurso periodístico, entre otros.

La importancia de considerar la variación lingüística en este tipo de estudios radica, precisamente, en el rol que cumple el conocimiento asociado no solo al contenido de los textos que se procesan, sino que también a sus características discursivas. No son pocos los psicolingüistas que destacan la relevancia del conocimiento previo en el establecimiento de la coherencia referencial (Anderson, Garrod y Sanford, 1983; Clifton y Ferreira, 1987; Crawley, 1986; Yecovich, Walker y Blackman, 1979; Gernsbacher, 1989). Incluso, Schwarz-Friesel (2007) sostiene que, en la resolución de pronombres, generalmente, el conocimiento previo y el dominio del contexto son más relevantes que las propias marcas textuales. En el caso del procesamiento del discurso en contextos especializados, el conocimiento previo puede tener impacto en el desempeño de la memoria de trabajo. De acuerdo a Ericsson y Kintsch (1995), existe un sistema de memoria que denominan la memoria de trabajo de largo plazo (MTLP), el cual entienden como un subcomponente de la memoria de largo plazo, hacia el cual se puede acceder directamente a partir de pistas en la memoria de trabajo de corto plazo. Este sistema permite una recuperación de la información de manera automática y rápida ya que la activación de nodos entre ambos tipos de memoria no estaría mediada por procesos conscientes. Kintsch, Patel y Ericsson (1999) sostienen que este sistema de me- 
moria solo es utilizado en procesamiento de alta especialización y exclusivamente por sujetos expertos.

A pesar de lo anteriormente expuesto, y también de la gran cantidad y variedad de estudios focalizados en el procesamiento de la coherencia referencial, al menos para el español, no existe uno que indague en este tipo de procesamiento en ámbitos especializados. Por esta razón, y considerando el supuesto que el establecimiento de la coherencia referencial no es universal e invariable y que, debido al tipo y cantidad de conocimiento involucrado, durante la lectura de textos académicos, el modo en que ciertos factores afectan su establecimiento es diferente a cómo lo hacen durante la lectura de textos no especializados; en este estudio se busca indagar en el efecto de la dirección y de la distancia referencial en el establecimiento de la coherencia referencial durante el procesamiento de textos académicos escritos en español.

\section{PROCESAMIENTO DE LA COHERENCIA REFERENCIAL Y LA DIRECCIÓN}

Según Gernsbacher (1990), la coherencia referencial puede ser entendida como la relación establecida entre una forma lingüística o expresión referencial y un concepto o antecedente, como en (1) "Juan Manuel cayó en el segundo asalto, no obstante, $\boldsymbol{s} \boldsymbol{e}$ puso de pie y terminó ganando el combate" o en (2) "Él sabía que podía ganar; sin embargo, el cansancio y la ansiedad llevaron al joven retador a la derrota". Asimismo, el establecimiento de esta relación puede ser de tipo anafórico, cuando la forma lingüística se presenta con posterioridad a la entidad a la cual hace referencia (como se aprecia en 1), o de tipo catafórico, cuando la forma lingüística se presenta con anterioridad (como se aprecia en 2). Ambos tipos constituyen manifestaciones de lo que entendemos como dirección referencial y han dado origen a una tradición de estudios lingüísticos y psicolingüísticos que se extiende por más de cuarenta años.

El procesamiento anafórico y su relación con otras variables han sido estudiados por diversos investigadores. Por ejemplo, Caramazza, Grober, Garvey y Yates (1977), a través de un estudio experimental, demostraron que la causalidad implícita de los verbos constituye una variable importante en la determinación de los antecedentes, potencialmente ambiguos, de pronombres anafóricos. Tales hallazgos han dado pie a una serie de estudios que profundizan en la relación entre la causalidad implícita y el procesamiento anafórico (Garnham, Oakhill, y Cruttenden, 1992; Cozijn, Commandeur, Vonk y Noordman, 2011; Koornneef y Sanders, 2013). Por otra parte, Garrod y Sanford (1977), interesados en relacionar el procesamiento anafórico y la distancia semántica, probaron que el procesamiento de oraciones anafóricas es más eficiente cuando se presenta primero una categoría 
(concepto general) y luego una instancia de dicha categoría (concepto específico). Así también, Gernsbacher (1989), interesada en relacionar el procesamiento anafórico y el grado de explicitud del mecanismo referencial, demostró que a mayor grado de explicitud, más eficiente es el procesamiento.

Por otra parte, en el caso del procesamiento catafórico, se destaca el trabajo de Gernsbacher y Shroyer (1989), quienes, interesados en estudiar el grado de accesibilidad de los conceptos durante la generación de una representación mental, demostraron que aquellos conceptos introducidos por la forma lingüística catafórica del inglés "this" son más accesibles para los sujetos que aquellos introducidos por el artículo indefinido " $a$ ". Según los autores, tal fenómeno tendría su explicación a partir del modelo de comprensión Structural Building Framework (Gernsbacher, 1990), específicamente, a partir de los procesos de reforzamiento y supresión, ya que los mecanismos catafóricos reforzarían la activación de ciertos conceptos, suprimiendo al mismo tiempo, la activación de otros. Otro trabajo destacable es el de Gernsbacher y Jescheniak (1995), quienes probaron que los mecanismos catafóricos fortalecen la permanencia de los conceptos en la representación mental de los oyentes. Según dichos autores, esto se debería a que los conceptos marcados catafóricamente se activan mucho más, suprimen de mejor forma la activación de otros conceptos y tienen mayor resistencia en ser suprimidos por otros. Por último, cabe mencionar el estudio de Van Gompel y Liversedge (2003), quienes interesados en la resolución de pronombres catafóricos, demostraron que la información morfológica se procesa con posterioridad al establecimiento de la relación referencial. Además, probaron que el procesamiento catafórico resulta más eficiente cuando existe congruencia de género y número.

Si bien existe una gran cantidad de trabajos enfocados en ambos tipos de dirección referencial, no existen muchos que comparen el procesamiento anafórico y el catafórico. Entre los escasos trabajos que han comparado el procesamiento de ambos tipos de dirección referencial, se encuentra la propuesta de Kennison, Fernández y Bowers (2009). Con el propósito de indagar en las posibles diferencias entre ambos tipos de procesamiento, a través de dos situaciones experimentales, los investigadores midieron tiempos de lectura durante el procesamiento de oraciones anafóricas y catafóricas en condiciones de congruencia e incongruencia de género entre un pronombre y un nombre propio. Los resultados demostraron diferencias en el procesamiento referencial, dependiendo de la dirección. Más específicamente, los resultados señalan que los pronombres anafóricos se resuelven más rápidamente que los pronombres catafóricos en la condición de congruencia de género. Además, los autores lograron comprobar que los comprendedores usan la información de congruencia de género de manera diferente, según el tipo de la dirección referencial que se procese, anafórica o catafórica. Esto debido a que se generan diferentes expectativas según sea el caso. 


\section{PROCESAMIENTO DE LA COHERENCIA REFERENCIAL Y LA DISTANCIA REFERENCIAL}

Otro rasgo de la coherencia referencial que ha sido frecuentemente considerado en diversos estudios es la distancia referencial (DR). Tal distancia constituye una medida meramente física, entendida como el espacio, en términos de cláusulas, entre la expresión referencial y su referente. Para Givón (1983, 1984, 1992), la DR puede ser asociada con dos fenómenos, la Accesibilidad y la Topicalidad, la primera entendida como una propiedad de los referentes anafóricos, relacionada con su grado de disponibilidad en una representación mental preexistente. La segunda se entiende como una propiedad de los referentes catafóricos y se relaciona con el grado de atención que se asigna al referente en la construcción de una nueva representación.

El alto grado de interés que ha concitado el procesamiento anafórico ha hecho que diversos especialistas pongan su atención en la accesibilidad, dando como resultado incluso teorías al respecto (Givón, 1983, 1984; Ariel, 1991, 2002, 2004). Tales teorías pretenden dar cuenta de los distintos grados de disponibilidad que pueden tener los referentes anafóricos durante el establecimiento de la coherencia referencial. El grado de accesibilidad se determina en virtud de la relación entre el grado de informatividad de la expresión referencial, y la distancia entre dicha expresión y el referente en el texto, en términos de cláusulas interpuestas. La teoría de la accesibilidad asume que a menor DR, mayor será la accesibilidad del concepto a recuperar, es decir, el referente estará más disponible en la memoria de quien procesa la información y por lo tanto, en tales ocasiones, se utilizarán expresiones referenciales con menor grado de informatividad. Por otra parte, se entiende que a mayor DR, el grado de accesibilidad es menor y, por lo tanto, en estos casos de baja continuidad referencial se utilizarán expresiones con mayor grado de informatividad.

Aunque según Givón (1984), la distancia solo debe ser concebida como una medida heurística para calcular el grado de accesibilidad referencial, no son pocos los especialistas que sostienen que esta sí tiene un rol importante en el procesamiento de pronombres (Maes y Noordman, 1995; Ariel, 1991; 2001). Gernsbacher (1990), por ejemplo, señala que, desde los estudios psicolingüísticos, existe suficiente evidencia para afirmar que la DR afecta el procesamiento. En su trabajo seminal, Clark y Sengul (1979) aportan a esta evidencia, demostrando que el procesamiento resulta más fácil si la expresión referencial se encuentra en la cláusula adyacente a la del referente. Más precisamente, estos autores hallaron que la accesibilidad en cláusulas adyacentes es mucho mayor que en condiciones de una o dos cláusulas de diferencia. Así también, utilizando el sistema de Eye Tracking, Ehrlich y Rayner (1983) llegaron a resultados similares. Estos hallazgos permiten sostener que si una entidad no ha sido mencionada por un tiempo, la activación de su 
representación mental se debilita, por lo que se hace menos accesible. De acuerdo a Gernsbacher (1990), cuando un referente no ha sido mencionado en un cierto lapso, existen diversas razones por las cuales puede hacerse menos accesible. Una de ellas es que su activación decae hasta desactivarse. Otra posible razón es que entre la expresión referencial y su referente aparezcan otros conceptos que puedan ser asociados con la expresión referencial, lo que sería plausible desde la noción de competencia de estímulos y procesamiento de información. Tal noción implica que diferentes estímulos compiten por ser más o menos relevantes en los procesos de codificación y almacenamiento (Richards y Krauter, 1999). Este último punto ha sido planteado como un argumento válido para explicar alteraciones en el procesamiento de información de naturaleza asociativa tanto en humanos (Vandorpe, De Houwer y Beckers, 2007; Boddez, Haesen, Baeyens y Beckers, 2014), como en animales (Shettleworth y Sutton, 2005; Bathellier, Tee, Hrovat y Rumpel, 2013).

A pesar de que la gran mayoría de los estudios que han considerado la distancia, lo ha hecho para estudiar el procesamiento anafórico, dejando de lado lo que sucede con el procesamiento catafórico, la revisión aquí presentada permite advertir que existe una gran cantidad de estudios y, en ese sentido, gran cantidad de conocimiento acumulado acerca de la coherencia referencial y de su procesamiento. Por supuesto, estos estudios han considerado diversos factores lingüísticos y psicolingüísticos, respondiendo, de este modo, a la necesidad de un trabajo interdisciplinario ya propuesta por Sanders y Gernsbacher (2004). No obstante lo anterior, y como ya se señaló al comienzo de este artículo, en la mayoría de estos estudios, el procesamiento se concibe como un fenómeno universal, invariable e independiente de las características de los textos (Garnham, Garrod y Sanford, 2006) y de las implicancias que tales diferencias podrían tener en el procesamiento.

\section{EXPERIMENTO 1}

El objetivo de este experimento fue determinar el efecto de la dirección y de la distancia referencial en el establecimiento de coherencia referencial durante el procesamiento de textos académicos escritos en español. Para ello se midieron los tiempos y la precisión de respuesta en el procesamiento de segmentos discursivos correspondientes a textos de Psicología y de Ingeniería en Construcción. La variable dirección se operacionalizó en términos de catáfora, cuando un pronombre era presentado previamente a su referente, y en términos de anáfora, cuando un pronombre era presentado con posterioridad. La variable distancia se operacionalizó en términos del espacio existente entre la cláusula que contenía la expresión referencial y la que contenía al referente. Así, si las cláusulas eran adyacentes, la distancia era entendida como cerca, mientras que si entre ambas existían una o dos cláusulas, era entendida como lejos (Givón, 1984). La combinación de las 
dos variables dio origen a cuatro condiciones experimentales, tal como se detalla en la Tabla II. Considerando los resultados de investigaciones previas y, al mismo tiempo, adoptando el supuesto de que el procesamiento de textos académicos fuera similar al procesamiento de textos que circulan en ámbitos generales, en este experimento se esperaban dos posibles tendencias en los resultados. Respecto de la dirección y de acuerdo al estudio de Kennison, Fernández y Bowers (2009), se podía esperar que el procesamiento de los pronombres anafóricos fuera más eficiente que el procesamiento de pronombres catafóricos. Respecto de la distancia, y según los resultados de los estudios de Clark y Sengul (1979) y Ehrlich y Rayner, (1983), se podía esperar que el procesamiento fuese más fácil cuando la expresión referencial se encontraba en la cláusula adyacente a la de su referente. Una diferencia en estas tendencias indicaría que el efecto de los factores dirección y distancia en el establecimiento de la coherencia referencial durante el procesamiento de textos académicos no es igual al efecto de estos factores durante el procesamiento de textos que circulan en contextos no especializados.

\subsection{Método}

\subsubsection{Participantes}

Inicialmente, en este estudio se evaluó a un total de treinta sujetos, de ellos seis fueron eliminados automáticamente usando como criterio la aleatoriedad de sus respuestas durante la fase de exposición (procesamiento) y de testeo (prueba). Dado que estos casos presentaron una alta aleatoriedad en sus tendencias de respuestas registradas (con un error estándar de la media alto y alejándose más de 3 D.T. de la media), se asumió que eran participantes no susceptibles de ser considerados en la condición experimental, siendo descartados del análisis (Nesselroade y Cattell, 2013; Harlow, Mulaik y Steiger, 2013; Elmes, Kantowitz y Roediger, 2011). Así, se obtuvo una muestra final de veinticuatro estudiantes universitarios de las carreras de Psicología $(n=12)$ y de Ingeniería en Construcción $(n=12)$. La distribución de los sujetos participantes en dos grupos pertenecientes a carreras diferentes obedeció a que, además de observar el procesamiento de textos en contextos académicos, pretendíamos indagar en posibles diferencias impuestas por disciplinas pertenecientes a ámbitos diferentes del conocimiento: Psicología como una disciplina de las ciencias sociales e Ingeniería en Construcción como una disciplina más cercana a las ciencias básicas. Todos los sujetos participantes pertenecían a cursos superiores a primer año, esto con el propósito de cautelar que tuvieran conocimientos disciplinares suficientes para enfrentar las temáticas abordadas en los textos experimentales. Por lo tanto, el rango de edad varió entre 19 y 25 años $(M=23,12 ; D T=1,31)$. La lengua materna de todos los sujetos era el 
español y su participación fue voluntaria, recibiendo una remuneración de $\$ 5.000$ (pesos chilenos).

\subsubsection{Materiales}

Tras un análisis de corpus desarrollado en el marco del proyecto FONDECYT 1120519, se determinó que el género discursivo prototípico de las carreras de Psicología y de Ingeniería en Construcción era el Manual. El macropropósito comunicativo de este género es instruir acerca de conceptos y/o procedimientos en una temática especializada; circula en el ámbito pedagógico y, preferentemente, tiene una organización discursiva de tipo descriptivo (Parodi, Venegas, Ibáñez y Gutiérrez, 2008). Se seleccionaron 24 fragmentos pertenecientes a este género, 12 correspondían a Psicología y 12 a Ingeniería en Construcción. En la selección de tales fragmentos se consideraron dos aspectos fundamentales: la dirección y la distancia referencial. De este modo, de los 12 fragmentos por cada disciplina, tres presentaban la condición anáfora-cerca, tres anáfora-lejos, tres catáfora-cerca, tres catáfora-lejos y, además, todos tenían una extensión entre 48 y 52 palabras. En la Tabla I se presentan ejemplos de los textos que debieron enfrentar los sujetos participantes. Para facilitar la visualización de la relación referencial, en esta oportunidad se destacan los elementos vinculados.

Tabla I. Textos de ejemplo según disciplina, dirección y distancia referencial.

\begin{tabular}{|l|l|l|l|}
\hline Disciplina & Dirección & $\begin{array}{l}\text { Distancia } \\
\text { Referencial }\end{array}$ & \multicolumn{1}{c|}{ Texto } \\
\hline Psicología & Catáfora & Cerca & $\begin{array}{l}\text { En la actualidad, cada vez más, las mujeres } \\
\text { pertenecientes a los diferentes niveles socioe- } \\
\text { conómicos, se integran al trabajo remunera- } \\
\text { do. Ello implica que los pequeños deban que- } \\
\text { dar al cuidado de éstas, sin embargo, no todas } \\
\text { las instituciones educacionales cuentan con } \\
\text { suficientes personas altamente calificadas para } \\
\text { atender todas las necesidades de los niños. (51 } \\
\text { palabras) }\end{array}$ \\
\hline $\begin{array}{l}\text { Ingeniería en } \\
\text { Construcción }\end{array}$ & Anáfora & Lejos & $\begin{array}{l}\text { Todas las mediciones llevan consigo errores } \\
\text { inevitables, por ello, entre los problemas más } \\
\text { frecuentes en el ámbito de la Topografía se en- } \\
\text { cuentran los trabajos requeridos para su com- } \\
\text { pensación. En este sentido, se realizan investi- } \\
\text { gaciones sobre la influencia que estos pueden } \\
\text { tener en la operación que se lleve a cabo. (49) } \\
\text { palabras) }\end{array}$ \\
\hline
\end{tabular}


Por último, cabe señalar que se utilizó el software E-Prime 2.0 Professional para diseñar y construir el instrumento experimental, lo que permitió medir los tiempos y la precisión de las respuestas emitidas por los sujetos participantes durante cada sesión experimental.

\subsubsection{Procedimiento}

Previo al desarrollo del experimento y después de firmar el respectivo consentimiento, a todos los participantes se les dio a conocer oralmente las instrucciones y se les asignó un equipo disponible del laboratorio para iniciar la rutina experimental. Cada participante realizaba el experimento de forma individual aislado visualmente del resto. La rutina experimental constaba de dos fases. La primera de estas tenía como propósito la familiarización con el software y la segunda, la ejecución de la tarea de resolución de pronombres. En la primera fase, los participantes, debían realizar un ejercicio a modo de prueba. Este ejercicio reunía las mismas características de la tarea descrita en la segunda fase, por lo tanto, con esta medida se aseguraba que los participantes pudieran ejecutarla adecuadamente. Una vez realizado dicho ejercicio y habiendo resuelto las posibles dudas de los participantes acerca de la tarea, se dio inicio a la segunda fase, la cual consistía en medir los tiempos y la precisión de las respuestas emitidas por cada sujeto participante. En esta fase, primero se les presentaba una diapositiva con una pregunta orientadora del tipo: "En el siguiente texto, ¿a qué hace referencia X?". Luego, a su propio ritmo, por medio de la técnica de lectura autocontrolada o self pacedreading (Bultena, Dijkstra y Van Hell, 2015) los sujetos participantes avanzaban a la siguiente diapositiva en la cual se presentaba el texto de Psicología o de Ingeniería en Construcción, de acuerdo a la disciplina a la cual pertenecían. Por último, una vez leído el texto, avanzaban hacia la siguiente diapositiva en la cual aparecían tres afirmaciones como respuestas alternativas y debían marcar solo aquella que consideraran correcta, presionando 1, 2 o 3 (Anexo 1). El orden en que aparecían los textos y las alternativas fue aleatorio. Además, cabe señalar que una vez que se avanzaba a la siguiente diapositiva, no era posible retornar a la información dispuesta en la diapositiva anterior. La sesión duró 45 minutos aproximadamente, tomando 10 minutos la primera fase y la segunda el tiempo restante.

Se utilizó un diseño factorial $2 \times 2$, en que se evaluó el efecto de la dirección (anafórica v/s catafórica) y la distancia (lejos v/s cerca). En la Tabla II se resumen

las cuatro condiciones experimentales resultantes para cada grupo (Psicología e Ingeniería en Construcción). 
Tabla II. Diseño experimental: distancia v/s dirección.

\begin{tabular}{|c|c|c|c|}
\hline \multicolumn{2}{|c|}{} & \multicolumn{2}{|c|}{$\begin{array}{c}\text { Distancia } \\
\text { (B) }\end{array}$} \\
\cline { 3 - 4 } \multicolumn{2}{|c|}{} & $\begin{array}{c}\text { Cerca } \\
\left(\mathrm{B}_{1}\right)\end{array}$ & $\begin{array}{c}\text { Lejos } \\
\left(\mathrm{B}_{2}\right)\end{array}$ \\
\hline \multirow{4}{*}{$\begin{array}{c}\text { Dirección } \\
(\text { A })\end{array}$} & $\begin{array}{c}\text { Anáfora } \\
\left(\mathrm{A}_{1}\right)\end{array}$ & $\begin{array}{c}\text { Anáfora - Cerca } \\
\left(\mathrm{A}_{1} \mathrm{~B}_{1}\right)\end{array}$ & $\begin{array}{c}\text { Anáfora - Lejos } \\
\left(\mathrm{A}_{1} \mathrm{~B}_{2}\right)\end{array}$ \\
\cline { 2 - 4 } & $\begin{array}{c}\text { Catáfora } \\
\left(\mathrm{A}_{2}\right)\end{array}$ & $\begin{array}{c}\text { Catáfora - Cerca } \\
\left(\mathrm{A}_{2} \mathrm{~B}_{1}\right)\end{array}$ & $\begin{array}{c}\text { Catáfora - Lejos } \\
\left(\mathrm{A}_{2} \mathrm{~B}_{2}\right)\end{array}$ \\
\hline
\end{tabular}

\subsection{Resultados}

En la Figura 1 se grafica el tiempo total (expresado en milisegundos o ms), que se demoraron los participantes $(n=24)$ en responder correctamente las preguntas asociadas a la rutina experimental según su carrera de procedencia (Psicología e Ingeniería en Construcción).

Los datos obtenidos revelan que tanto el efecto simple de la distancia como el de la dirección sobre la eficacia de los participantes en resolver cada tarea de lectura resultaron estadísticamente significativos. En el caso de la dirección (catáforaanáfora), se observó que las catáforas son procesadas más eficientemente que las anáforas $(F[3,20]=131,112 ; \mathrm{p} \leq 0,001$, partial $\eta 2=0,684)$ durante la sesión experimental. De esta manera, se puede afirmar que el establecimiento de la coherencia referencial se facilita en la presencia de catáforas (Media $=3221,9741$ y 3387,4731 ms.; $D T=1653,92631$ y 2351,35718 ms. respectivamente) y se dificulta en el caso de las anáforas (Media $=5213,3412$ y $4722,0032 \mathrm{~ms}$, DT $=3373,40188$ y $3419,02451 \mathrm{~ms})$. En el caso de la dirección, se observó un efecto significativo $(F[3,20]=22,201 ; p=0,003$, partial $\eta 2=0,049)$, de forma tal que en casos de distancia referencial cerca, el procesamiento fue más eficiente que en aquellos casos en que la distancia referencial correspondía a lejos. En este sentido, la distancia constituye un facilitador (cuando están cerca) o un dificultador (cuando están lejos) de ambos procesos. También se observó una interacción significativa entre la dirección y la distancia $(F[3,20]=19,416 ; p=0,009, \eta 2=0,603)$. Como resultado de la interacción existente entre la dirección (anáfora-catáfora) y la distancia (cerca-lejos) y tal como se demuestra en la Figura 1, se observó que independiente de la carrera de procedencia ( $\operatorname{ts}[22] \leq-1,066$; ps $\geq 0,295$ ), el establecimiento de la coherencia referencial es más eficiente en el caso de las catáforas cerca, seguido por las catáforas lejos y así hasta llegar a la anáfora lejos. 
RLA. Revista de Lingüística Teórica y Aplicada, 53 (2), II Sem. 2015

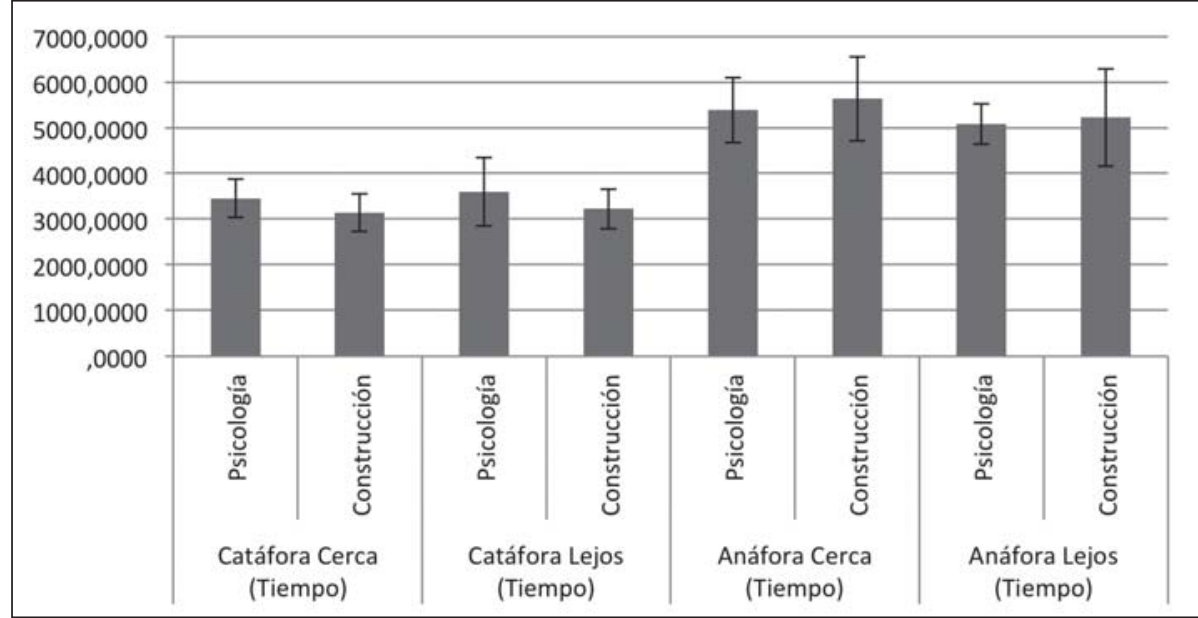

Figura 1. Tiempo de respuesta según condición experimental.

El eje y (ordenada) representa el tiempo de respuesta correcta en milisegundos emitido por cada participante. El eje x (abscisa) corresponde a las cuatro condiciones experimentales comparadas según carrera de procedencia. Las barras de error representan el error estándar de la media de milisegundos emitidos por cada grupo.

\subsection{Discusión}

Los resultados obtenidos demuestran que la variable distancia tiene un efecto significativo en el establecimiento de la coherencia referencial durante el procesamiento de textos académicos escritos en español, es decir, la distancia incide en el procesamiento de pronombres, en tanto se probó que cuando existe distancia referencial cerca, el procesamiento resulta más rápido. Por lo tanto, los resultados son consistentes con el trabajo de Clark y Sengul (1979) y el de Ehrlich y Rayner (1983). Esto quiere decir que, durante el procesamiento de textos académicos, la variable distancia presenta el mismo comportamiento que tiene cuando se procesan textos no especializados.

Según Gernsbacher (1990), la razón de por qué el procesamiento resulta más rápido cuando existe distancia referencial cerca se debe a que no habría conceptos nuevos intervinientes que debiliten la activación del concepto referido, de modo que este se mantendría accesible en la representación mental de quien procesa la información. Otra razón sería el lugar privilegiado que tendría en la memoria de trabajo aquella cláusula que se encuentra más cerca de la cláusula en que se pre- 
senta el pronombre. Esto pues, según Clark y Sengul (1979), el referente que se presenta en aquella cláusula resulta más fácil de captar que aquel que se presenta dos o tres cláusulas más lejanas. Esto podría ser explicado bajo la noción de saliencia o relevancia biológica de la información (Lavis, Kadib, Mitchell y Hall, 2011). Desde esta mirada, la cercanía de la cláusula actuaría como un facilitador asociativo del procesamiento y codificación de cada cláusula, facilitando así su almacenaje tanto en la memoria de trabajo como en la de largo plazo. El efecto acá descrito ha sido ampliamente documentado en tareas asociativas de reevaluación retrospectiva de estímulos (Le Pelley y McLaren, 2001; Vadillo, Castro, Matute y Wasserman, 2008; Jamieson y Chubala, 2012).

Por otra parte, respecto de la variable dirección los resultados demuestran que también tiene un efecto significativo en el establecimiento de coherencia referencial durante el procesamiento de textos académicos escritos en español, específicamente, en la condición catafórica. Al presentarse pronombres catafóricos, los participantes de ambas disciplinas lograron un procesamiento más rápido y con mayor precisión que con pronombres anafóricos. Estos resultados serían inconsistentes con los obtenidos por Kennison, Fernández y Bowers (2009), en cuyo trabajo los pronombres anafóricos fueron procesados más eficientemente. Sin embargo, refuerzan los planteamientos de Gernsbacher y Shroyer (1989), Gernsbacher y Jescheniak (1995) acerca de la mayor accesibilidad que los mecanismos catafóricos proporcionarían. La explicación para esta diferencia -a juicio de los autores del presente estudio- es que estos resultados se deben a que los participantes de ambas disciplinas pertenecían a cursos superiores a primer año, por lo tanto, dominaban conocimientos específicos del área disciplinar. En este sentido, tal conocimiento pudo haber permitido que generaran mayores expectativas acerca de los conceptos referidos y que les resultara más fácil la desambiguación de los pronombres referidos a ellos.

A partir de estos resultados, además de demostrar que la variable dirección incide en el procesamiento de pronombres, se evidencia que existe una diferencia en el procesamiento de textos académicos y no especializados. La variable dirección al enfrentar textos académicos, presentó un comportamiento diferente de aquel que se manifiesta cuando se enfrentan textos no especializados. Tal diferencia tendría su explicación en el posible efecto de la variable conocimiento disciplinar en el procesamiento de textos académicos, el cual no solo incluye conocimientos asociados a contenidos, sino que también a las formas discursivas en que el conocimiento disciplinar se construye y comunica. El discurso académico presenta características particulares, pues según Hyland (2000), evidencia la forma en que se construye, se evalúa y se negocia conocimiento dentro de una comunidad discursiva específica, por lo tanto, se puede esperar que quienes enfrentan este tipo de discurso desarrollen ciertas habilidades para lograr su comprensión y posean conocimiento acerca de aquellas formas propias de sus disciplinas. Por otra parte, respecto de la 
interacción de las variables distancia y dirección, y tal como se observa en la Figura 1, se genera un mayor efecto sobre el tiempo y la precisión en que los textos son procesados cuando ambas se presentan en la condición catáfora-cerca. En tal condición, el procesamiento es más eficiente que en cualquier otra, resultando ser el contexto que más facilita el procesamiento de pronombres durante la lectura de textos académicos escritos en español. Por último, la condición anáfora-lejos es la que más dificultaría dicho procesamiento. A partir de los resultados aquí expuestos, se determinó que ambas variables y la interacción de estas tienen un efecto importante en el establecimiento de la coherencia referencial cuando se procesan textos académicos escritos en español.

Dado este contexto, parece necesario indagar en el rol de la variable conocimiento disciplinar en el establecimiento de la coherencia referencial durante la lectura de textos académicos escritos en español. Para ello, se llevó a cabo un segundo experimento, en el cual se pretendió determinar el efecto de la variable conocimiento disciplinar y su interacción con la variable dirección en el establecimiento de la coherencia referencial durante el procesamiento de textos académicos. Cabe señalar que en este segundo experimento no se tomó en consideración la variable distancia, debido a que ha quedado de manifiesto que incide en el procesamiento de textos académicos de la misma forma que en el procesamiento de textos no académicos, por consiguiente y siguiendo las interrogantes que motivaron este trabajo, resultaba irrelevante observar nuevamente su incidencia e interacción con las variables en cuestión.

\section{EXPERIMENTO 2}

El propósito de este experimento fue determinar la interacción de las variables conocimiento disciplinar y dirección en el establecimiento de coherencia referencial durante el procesamiento de textos académicos escritos en español. Para ello, al igual que en el experimento 1 , se midieron los tiempos y la precisión de las respuestas durante el procesamiento de textos correspondientes a textos de Psicología y de Ingeniería en Construcción. La variable dirección se operacionalizó de la misma forma que en el experimento 1. Mientras que la variable conocimiento disciplinar se operacionalizó en términos de congruencia e incongruencia. La primera consistía en que los participantes debían enfrentar textos académicos escritos en español correspondientes a su área disciplinar y en la segunda, debían enfrentar textos que no correspondían a su área disciplinar. Así, la combinación de las dos variables dio origen a cuatro condiciones experimentales, tal como se detalla en la Tabla III. En este experimento se esperaba que, por una parte, en la condición de congruencia disciplinar se mantuvieran los resultados obtenidos en el experimento 1 , pues si el conocimiento disciplinar incidiera en el procesamiento, debería 
replicarse el efecto de la catáfora. Por otra parte, respecto de la condición de incongruencia disciplinar, se esperaba que los pronombres anafóricos se procesaran más eficientemente (Kennison, Fernández y Bowers, 2009), ya que en ausencia de conocimiento disciplinar, a los participantes les resultaría más difícil crear expectativas acerca de un concepto referido catafóricamente y su desambiguación les resultaría más compleja, por lo tanto podría tomarles más tiempo la resolución.

\subsection{Método}

\subsubsection{Participantes}

Siguiendo los mismos criterios de filtraje de participantes expresados en el experimento 1, en este estudio participaron veinticinco estudiantes de las carreras de Psicología $(n=13)$ y de Ingeniería en Construcción $(n=12)$, cuyas características fueron similares a la de los participantes del experimento 1 . El rango de edad varió entre 18 y 27 años $(M=24,81$; DT=2,06) y su participación también fue remunerada con $\$ 5.000$ (pesos chilenos).

\subsubsection{Materiales}

Se usaron los mismos materiales creados para el experimento 1 . Es decir, se consideraron los 24 fragmentos de manuales, 12 de Psicología y 12 de Ingeniería en Construcción, todos con las mismas condiciones descritas en el experimento 1.

\subsubsection{Procedimiento}

Los participantes se enfrentaron a la misma rutina experimental descrita en el experimento 1, pero en esta oportunidad se incluyó la variable conocimiento disciplinar con sus valores congruencia e incongruencia. Los participantes debieron enfrentar seis textos de su disciplina y otros seis textos correspondientes a una disciplina diferente de la suya. Así, quienes pertenecían a la disciplina de Psicología enfrentaron textos de Psicología e Ingeniería en Construcción y quienes pertenecían a la disciplina de Ingeniería en Construcción, textos de Ingeniería en Construcción y Psicología. En este experimento, al igual que en el anterior, se realizaron dos fases, la de familiarización con el software y la de ejecución de la tarea de resolución de pronombres. Por lo tanto, hubo un ejercicio de prueba y, luego, se midieron los tiempos y la precisión de las respuestas. La sesión duró 50 minutos aproximadamente, distribuidos en 10 minutos para la primera fase de familiarización y 40 minutos para la fase experimental principal. 


\subsubsection{Diseño y plan de análisis}

Tal como se observa en la Tabla III y al igual que en el experimento 1, se utilizó un diseño factorial 2x2. En este diseño se evaluó el efecto de la dirección (anafórica $\mathrm{v} / \mathrm{s}$ catafórica) y del conocimiento disciplinar (congruencia v/s incongruencia).

Tabla III. Diseño experimental: dirección v/s conocimiento disciplinar.

\begin{tabular}{|c|c|c|c|}
\hline & & \multicolumn{2}{|c|}{ Conocimiento disciplinar (B) } \\
\hline & & $\begin{array}{l}\text { Congruencia } \\
\left(\mathrm{B}_{1}\right)\end{array}$ & $\begin{array}{c}\text { Incongruencia } \\
\left(\mathrm{B}_{2}\right)\end{array}$ \\
\hline \multirow{2}{*}{$\begin{array}{l}\text { Dirección } \\
\text { (A) }\end{array}$} & $\begin{array}{c}\text { Anáfora } \\
\left(\mathrm{A}_{1}\right)\end{array}$ & $\begin{array}{c}\text { Anáfora - congruente } \\
\left(A_{1} B_{1}\right)\end{array}$ & $\begin{array}{c}\text { Anáfora - incongruente } \\
\qquad\left(A_{1} B_{2}\right)\end{array}$ \\
\hline & $\begin{array}{c}\text { Catáfora } \\
\left(\mathrm{A}_{2}\right)\end{array}$ & $\begin{array}{l}\text { Catáfora - congruente } \\
\qquad\left(\mathrm{A}_{2} \mathrm{~B}_{1}\right)\end{array}$ & $\begin{array}{l}\text { Catáfora - incongruente } \\
\qquad\left(\mathrm{A}_{2} \mathrm{~B}_{2}\right)\end{array}$ \\
\hline
\end{tabular}

\subsection{Resultados}

En la Figura 2 se grafica el tiempo total (expresado en milisegundos o ms), que se demoran los participantes $(n=25)$ en responder correctamente las preguntas asociadas a la rutina experimental, según carrera de procedencia (Psicología e Ingeniería en Construcción). Nuevamente se observó un efecto principal de la dirección catáfora $\mathrm{v} / \mathrm{s}$ anáfora $(\mathrm{F}[1,20]=132,326 ; \mathrm{p}=0,005$; partial $\eta 2=0,591)$, donde las catáforas son procesadas más eficientemente que las anáforas. De igual modo, se observó un efecto principal de la congruencia disciplinar (congruente v/s incongruente), donde los textos congruentes fueron procesados más eficientemente que los incongruentes $(F[3,20]=4,890 ; p=0,042$; partial $\eta 2=0,471)$. De igual modo, se observó un efecto de interacción significativa entre ambos factores $(F[3,20]=$ $14,331 ; \mathrm{p}=0,022, \eta 2=0,563)$. De modo que las catáforas congruentes eran procesadas más eficientemente que las catáforas incongruentes y así hasta llegar a las anáforas incongruentes, las que fueron procesadas más ineficientemente. 


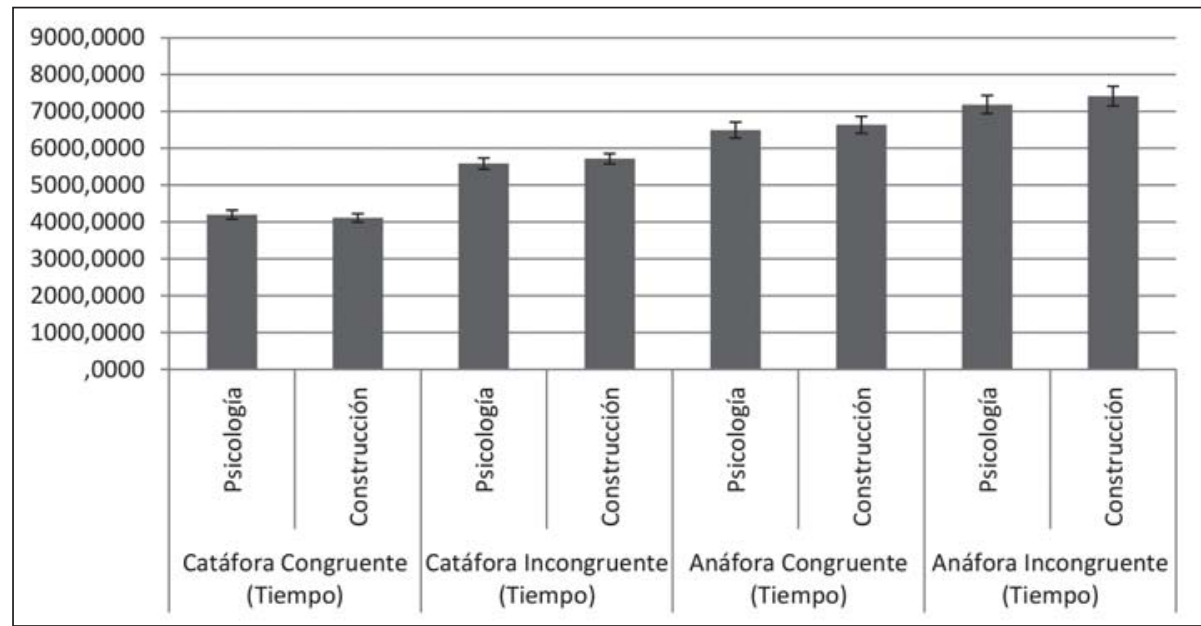

Figura 2. Tiempo de respuesta en condiciones experimentales de congruencia e incongruencia disciplinar.

El eje y (ordenada) representa el tiempo de respuesta en milisegundos emitido por cada participante. El eje x (abscisa) corresponde a las cuatro condiciones experimentales comparadas según carrera de procedencia en condiciones de congruencia e incongruencia disciplinar. Las barras de error representan el error estándar de la media de milisegundos emitidos por cada grupo.

\subsection{Discusión}

Los resultados obtenidos revelan que la variable conocimiento disciplinar tiene un efecto significativo en el establecimiento de la coherencia referencial durante el procesamiento de textos académicos escritos en español. Esto pues, demostró que el procesamiento resultó de manera más rápida y precisa cuando los participantes debieron enfrentar textos académicos de su propia disciplina, es decir, en la condición de congruencia disciplinar. Además, los resultados dan cuenta de que el procesamiento de textos académicos alcanzó mayor eficiencia, precisamente, cuando los participantes debieron enfrentar pronombres catafóricos. De este modo, se comprueba que el conocimiento disciplinar es una variable que tiene un efecto importante en el procesamiento de textos académicos y que su interacción con la variable dirección catáfora es la condición que mayormente facilita el procesamiento de tales textos. A partir de estos resultados y al observar que se replicaron los datos obtenidos en el experimento 1, es factible suponer que el conocimiento disciplinar que poseían los participantes permitió una desambiguación de pronombres mucho más eficiente. El efecto del conocimiento disciplinar puede expli- 
carse por medio del sistema de MTLP, propuesto por Ericsson y Kintsch (1995), en tanto que tratándose de sujetos expertos, serían capaces de hacer uso de este sistema de memoria, lo que les permitiría recuperar información de manera más eficiente al momento de establecer relaciones de coherencia referencial.

Por otra parte, respecto de la condición de incongruencia disciplinar, es decir, cuando los participantes debieron enfrentar textos que no eran de su área disciplinar, nuevamente resultó más eficiente el procesamiento de pronombres catafóricos. Esto llama la atención, pues se esperaba que ante la ausencia de conocimiento disciplinar los pronombres catafóricos fueran más difíciles de procesar, pues el grado de expectativas sería menor y la desambiguación de los conceptos resultaría más compleja. Estos resultados podrían revelar, por una parte, que existiría una supremacía de la catáfora en los textos académicos, es decir, existiría mayor frecuencia de mecanismos catafóricos que anafóricos. Por lo tanto, a los participantes les resultó más fácil procesar en la condición catafórica, ya que constantemente enfrentan este tipo de mecanismos en los textos de sus disciplinas. Por otra parte, al igual como demuestran los estudios de Gernsbacher y Shroyer (1989) y Gernsbacher y Jescheniak (1995), estos resultados indicarían que cuando se trata de textos que circulan en contextos especializados, los conceptos marcados catafóricamente tendrían mayor accesibilidad, se activarían mucho más, suprimirían la activación de otros conceptos de mejor forma y resistirían de manera más adecuada la activación de otros conceptos intervinientes.

Sin embargo, a pesar de que en la condición de incongruencia disciplinar los resultados fueron diferentes de los esperados, a través de este experimento queda en evidencia, al menos en la condición de congruencia disciplinar, que el procesamiento de textos académicos es diferente de aquel que se lleva a cabo a partir de textos que circulan en contextos no especializados.

\section{DISCUSIÓN GENERAL}

En primer lugar, los resultados del experimento 1 ratifican los hallazgos de Clark y Sengul (1979), así como también los de Ehrlich y Rayner (1983), respecto de la distancia referencial (DR), puesto que en condición cerca se procesó de manera más eficiente que en condición lejos. En este caso, el establecimiento de coherencia referencial durante la lectura de textos académicos no probó ser diferente a la lectura de textos no especializados. Esto se podría deber a que la desambiguación en casos de adyacencia se lleva a cabo por medio de expresiones con menor grado de informatividad, lo que implica un conocimiento principalmente lingüístico y no de expresiones particulares de una disciplina.

En este mismo experimento, por otra parte, los resultados respecto de la variable dirección son inconsistentes con hallazgos anteriores. En el presente estudio, 
a diferencia de lo que sucedió en el trabajo de Kennison, Fernández y Bowers (2009), el establecimiento de la coherencia referencial es más eficiente en casos de catáfora. Considerando que los materiales utilizados por los autores antes aludidos contemplaban solo oraciones aisladas de discurso general, es posible sostener que, en el caso de la dirección, el establecimiento de la coherencia referencial durante la lectura de textos académicos es diferente a la de textos no especializados. En este caso se puede suponer que la razón para tales resultados fue que cuando los participantes tenían que procesar información en la condición catafórica, a causa de su conocimiento de la disciplina, ellos no solo generaron mayores expectativas (Gernsbacher, 1990), sino que también podían acceder al concepto referido con mayor certeza (Ericsson y Kintsch, 1995) y, de este modo, de manera más eficiente. Un ejemplo de lo anterior es lo que sucede con el texto: "Se habla de él, sobre todo, por su falta de dogmatismo; por su inclinación a admitir sucesivos replanteos de un mismo problema y, a diferencia de Platón, porque reconoce la existencia de dificultades. Por esto, Aristóteles ha sido admirado por diversos filósofos como es el caso de James, en Oxford". (PSI - Catáfora - Lejos, 50). En este texto, que forma parte de los materiales utilizados, se presenta el pronombre él, tercera persona singular del masculino, y luego en el cotexto, aparece información relacionada con el tópico, como "su falta de dogmatismo", "a diferencia de Platón" y "reconoce la existencia de dificultades", información que facilitará la desambiguación del pronombre a un miembro de la comunidad disciplinar, que maneje información respecto de Aristóteles.

Debido, precisamente, a la importancia que se le atribuyó en los resultados asociados a la condición catafórica, se llevó a cabo el segundo experimento, en el que el conocimiento de la disciplina se planteó como una de las variables. En este caso, la importancia del conocimiento disciplinar para el establecimiento de la coherencia referencial durante el procesamiento de textos académicos quedó comprobada ya que fue posible determinar que la coherencia referencial se establecía de manera más eficiente cuando los participantes conocían el tema acerca del cual trataban los textos, es decir en condición de congruencia disciplinar y no de incongruencia.

No obstante lo anterior, y contrario a lo esperado, los resultados demostraron que las catáforas en condición de incongruencia disciplinar siguieron siendo procesadas de manera más eficiente que las anáforas en condición de congruencia disciplinar, es decir, independiente del grado de conocimiento disciplinar de los participantes, ellos procesaban las catáforas de manera más eficiente. En este punto, es oportuno mencionar lo que Kennison, Fernández y Bowers (2009) plantean respecto de sus resultados. Ellos argumentan que la resolución más eficiente de las anáforas se debía a que las expectativas por encontrar un nombre seguido por un pronombre son mayores que las expectativas por encontrar un pronombre seguido de un nombre debido a la frecuencia con que esta forma aparece en los textos. 
En el presente estudio se podría utilizar un argumento similar, aludiendo a que probablemente en el discurso académico, las catáforas no son tan escasas como en el discurso general y ya que los miembros de una comunidad disciplinar particular utilizan el discurso de tal comunidad adoptando sus características formales y funcionales, los participantes del estudio procesarían las catáforas de manera más eficiente. Sin embargo, para utilizar tal argumento, es absolutamente necesario desarrollar un estudio de corpus detallado, en el que se describa la coherencia referencial en el discurso académico, no solo en cuanto a la dirección, sino que también en cuanto a los tipos de mecanismos lingüísticos utilizados para guiar el establecimiento de la coherencia referencial.

\section{CONCLUSIONES}

La motivación principal del estudio presentado en este artículo surge del supuesto de que el establecimiento de la coherencia referencial está mediado por el contexto y que, por lo tanto no se trata de un fenómeno invariable, en consecuencia, no se lleva a cabo de la misma manera cuando se procesa discurso especializado, que cuando se procesa discurso no especializado. Tal supuesto se basa en descripciones lingüísticas que demuestran diferencias no solo entre el discurso académico y el discurso general, sino que también entre el discurso académico que se utiliza en disciplinas diferentes (Bhatia, 2004; Hyland, 2004, 2008; Biber, 2006; Ibáñez, 2010). Con esta forma de abordar el fenómeno no se ha pretendido abandonar al sujeto como centro de interés en el procesamiento lingüístico, pero sí, en concordancia con lo planteado por Garnham, Garrod y Sanford (2006), también se ha puesto atención a los efectos que el contexto real de uso pueda imponer. En este sentido, y reconociendo la relevancia del conocimiento previo en el procesamiento de la coherencia referencial (Caramazza, Grober, Garvey y Yates, 1977; Anderson, Garrod y Sanford, 1983; Stevenson y Vitkovitch, 1986; Schwarz-Friesel, 2007), se entiende que cuando se trata de procesar discurso académico, tal conocimiento no solo incluye el conocimiento de mundo, sino que también el relacionado con la disciplina en cuestión y con las formas discursivas que en tal disciplina se construye y transmite el conocimiento.

Si bien los resultados obtenidos en el estudio aportan datos empíricos para reafirmar resultados previos acerca del establecimiento de la coherencia referencial, algunos de ellos también han permitido comprobar que en ciertos aspectos el discurso académico se procesa de forma diferente al discurso no especializado. Esta última aseveración es posible a partir de la comparación de los resultados del estudio efectuado con otros, en los que para construir los materiales se utilizaron textos no especializados o en los que no se consideraba el contexto de circulación por tratarse de pares de oraciones aisladas. Por otra parte, estos resultados también 
permiten afirmar que el conocimiento previo asociado a la disciplina sí tiene un rol importante en el establecimiento de la coherencia referencial.

A partir de estos resultados, un estudio de corpus cobra mayor sentido, pues diversos lingüistas interesados en describir la variación del discurso sostienen que el discurso que circula en un determinado ámbito académico, profesional o científico mantiene ciertas características, (Bhatia, 2004; Biber, 2006; Biber, Connor y Upton, 2007; Ibáñez, 2010) no solo funcionales, sino que también formales, que permiten distinguirlo de otros. Además, señalan que tales características discursivas son adoptadas y utilizadas por los miembros de una comunidad en la construcción y transmisión del conocimiento disciplinar. Este tipo de estudios no se limita a aquellos que describen la organización general de los textos (Swales, 1990, 2004) o los que describen rasgos lexicogramaticales (Halliday y Martin, 1993), sino que también se extiende a aquellos que describen la coherencia referencial. Un ejemplo de aquellos trabajos es el realizado por Swanson (2003), quien da cuenta de las diferencias en términos de coherencia referencial entre los géneros revistas, relatos y textos académicos.

En este sentido, por medio de este trabajo, ha sido posible comprobar que dado que la coherencia corresponde a una representación mental generada, en gran medida, a partir de la información presente en un texto y que, en ese sentido, su establecimiento está guiado por las características de los textos (Kintsch, 1988; Van den Broek, 1994; Gernsbacher y Givón, 1995; Graesser, Millis, y Zwaan, 1997; Sanders y Spooren, 2001), tal representación, así como el proceso a partir del cual es generada varían de acuerdo a las características del género o del tipo de discurso al cual el texto que se procesa pertenece.

\section{REFERENCIAS}

Anderson, A., Garrod, S., y Sanford, A. (1983). The accessibility of pronominal antecedents as a function of episode shifts in narrative texts. The Quarterly Journal of Experimental Psychology Section A: Human Experimental Psychology, 35(3), 427-440.

Ariel, M. (1991). The function of accessibility in a theory of grammar. Journal of Pragmatics, 12, 5/6, 567-600.

Ariel, M. (2001). Accessibility theory: an overview. En T. Sanders, J. Schilperoord y W. Spooren (Eds.), Text representation: linguistic and psycholinguistic aspects (pp. 29-87). Amsterdam: John Benjamins.

Ariel, M. (2002). The possessive NP construction: Discourse function and discourse profile. Berkeley Linguistics Society, 28, 15-26.

Ariel, M. (2004). Accessibility Marking: Discourse Functions, Discourse Profiles, and Processing Cues. Discourse Processes, 37(2), 91-116. 
Ariel, M. (2007). A grammar in every register? The case of definite descriptions. En Nancy Hedberg y Ron Zacharsky (Eds.), The Grammar-Pragmatics Interface: Essays in Honor of Jeanette K. Gundel (pp. 265-293). Amsterdam: John Benjamins.

Bathellier, B., Tee, S. P., Hrovat, C., y Rumpel, S. (2013). A multiplicative reinforcement learning model capturing learning dynamics and interindividual variability in mice. Proceedings of the National Academy of Sciences, 110(49), 19950-19955.

Bhatia, V. K. (2004). Worlds of written discourse: A Genre-based view. Londres: Bloomsbury.

Biber, D. (2006). University language: A corpus-based study of spoken and written registers. Amsterdam: John Benjamins.

Biber, D., Connor, U., y Upton, T. A. (2007). Discourse on the move. Using corpus analysis to describe discourse structure. Amsterdam: John Benjamins.

Boddez, Y., Haesen, K., Baeyens, F., y Beckers, T. (2014). Selectivity in associative learning: a cognitive stage framework for blocking and cue competition phenomena. Frontiers in psychology, 5(135), 1-13.

Bultena, S., Dijkstra, T., y Van Hell, J. G. (2015). Language switch costs in sentence comprehension depend on language dominance: Evidence from self-paced reading. Bilingualism: Language and Cognition, 18(03), 453-469.

Caramazza, A., Grober, E., Garvey, C., y Yates, J. (1977). Comprehension of Anaphoric Pronouns. Journal of Verbal Learning and Verbal Behavior, 16, 601-609.

Clark, H. H. y Sengul, C. J. (1979). In search of referents for nouns and pronouns. Memory and Cognition, 7, 35-41.

Clifton, C. y Ferreira, F. (1987). Modularity in sentence comprehension. En J. Garfield, (Ed.) Modularity in knowledge representation and natural language processing, (pp. 277-290). Cambridge, MA: MIT Press.

Cozijn, R., Commandeur, E., Vonk, W., y Noordman, L. (2011). The time course of the use of implicit causality information in the processing of pronouns: A visual world paradigm study. Journal of Memory and Language, 64(4), 381-403.

Crawley, R. (1986). Some factors influencing the comprehension of pronouns. En C. Clifton (Ed.), Proceedings of the Eighth Annual Conference of the Cognitive Science Society (pp. 613-620). Hillsdale, NJ: Lawrence Erlbaum Associates.

Ehrlich, K. y Rayner, K. (1983). Pronoun assignment and semantic integration during reading: Eye movement and immediacy of processing. Journal of Verbal Learning and Verbal Behavior, 22(1), 75-87.

Elmes, D., Kantowitz, B., y Roediger III, H. (2011). Research methods in psychology. Cengage Learning.

Ericsson, K. A., y Kintsch, W. (1995). Long-term working memory. Psychological Review, 102(2), 211.

Garnham, A. (2001). Mental models and the interpretation of anaphora. Hove, East 
Sussex: Psychology Press.

Garnham, A., Oakhill, J. V., y Cruttenden, H. (1992). The role of implicit causality and gender cue in the interpretation of pronouns. Language and Cognitive Processes, 7(3-4), 231-255.

Garnham, A., Garrod, S., y Sanford, A. (2006). Observations on the past and future of psycholinguistics. En M. Traxler y M. Gernsbacher (Eds.), Handbook of Psycholinguistics (pp. 1-18). Amsterdam: Elsevier/Academic Press.

Garrod, S. y Sanford, A. (1977). Interpreting Anaphoric relations: The Integration of Semantic Information while Reading. Journal of Verbal Learning and Verbal Behavior, 16(1), 77-90.

Gernsbacher, M. A. (1989). Mechanisms that improve referential access. Cognition, 32(2), 99-156.

Gernsbacher, M. A. (1990). Language Comprehension as Structure Building. Oregon: University of Oregon.

Gernsbacher, M. A. y Shroyer, S. (1989). The cataphoric use of the indefinite this in spoken narratives. Memory \& Cognition, 17, 536-540.

Gernsbacher, M. A. y Givón, T. (1995). Coherence in spontaneous text. Philadelphia, PA: John Benjamins.

Gernsbacher, M. A. y Jescheniak, J. D. (1995). Cataphoric devices in spoken discourse. Cognitive Psychology, 29(1), 24-58.

Givón, T. (1983) Topic Continuity in Discourse: A quantitative cross-language study. Amsterdam: John Benjamins.

Givón, T. (1984). Syntax: A functional-typological introduction. Vol. 1. Amsterdam: John Benjamins.

Givón, T. (1992). The Grammar of Referential Coherence as Mental Processing Instructions. Linguistics, 30(1), 5-56.

Graesser, A. C., Millis, K. K., y Zwaan, R. A. (1997). Discourse comprehension. Annual Review of Psychology, 48, 163-189.

Halliday, M. (1994). Introduction to Functional Grammar. London: Arnold.

Halliday, M. y Martin, J. (1993). Writing Science, Literacy and Discoursive Power. Londres: The Falmer Press.

Harlow, L. L., Mulaik, S. A., y Steiger, J. H. (2013). What if there were no significance tests? Psychology Press.

Hyland, K. (2000). Disciplinary discourses: social interactions in academic writing. Harlow: Pearson Education Ltd.

Hyland, K. (2004). Disciplinary interactions: metadiscourse in L2 postgraduate writing. Journal of Second Language Writing, 13(2), 133-151.

Hyland, K. (2008). Genre and academic writing in the disciplines. Language Teaching, 41(4), 543-562.

Ibáñez, R. (2010). The Disciplinary Text genre as a means for accessing disciplinary knowledge: A study from a Genre Analysis perspective. En G. Pa- 
rodi (Ed.), Discourse genres in Spanish: Academic and professional connections (pp.189-211). Amsterdam: John Benjamins.

Jamieson, R. K., y Chubala, C. M. (2012). A Memory-Based Explanation of Higher-Order Retrospective Revaluation. Canadian journal of experimental psychology-revue canadienne de psychologie experimentale, 66(4), 268-327.

Kennison, S., Fernández, E. y Bowers, J. (2009). Processing differences for anaphoric and cataphoric pronouns: Implications for theories of referential processing. Discourse Processes, 46, 25-35.

Kintsch, W. (1988). The use of knowledge in discourse processing: A construction-integration model. Psychological Review, 95, 163-182.

Kintsch, W., Patel, V. L., y Ericsson, K. A. (1999). The role of long-term working memory in text comprehension. Psychologia, 42(4), 186-198.

Koornneef, A. y Sanders, T. (2013). Establishing coherence relations in discourse: The influence of implicit causality and connectives on pronoun resolution. Language \& Cognitive Processes, 28(8), 1-38.

Lavis, Y., Kadib, R., Mitchell, C., y Hall, G. (2011). Memory for, and salience of, the unique features of similar stimuli in perceptual learning. Journal of Experimental Psychology: Animal Behavior Processes, 37(2), 211.

Le Pelley, M. E., y McLaren, I. P. L. (2001). Retrospective revaluation in humans: Learning or memory? The Quarterly Journal of Experimental Psychology: Section $B, 54(4), 311-352$.

Maes, A. y Noordman, L. (1995). Demonstrative nominal anaphors: a case of non identificational markedness. Linguistics, 33(2), 255-282.

Nesselroade, J. R., y Cattell, R. B. (Eds.). (2013). Handbook of multivariate experimental psychology. Springer Science \& Business Media.

Parodi, G., Venegas, R., Ibáñez, R. y Gutiérrez, R. (2008). Géneros Académicos y Géneros Profesionales: Accesos discursivos para saber y hacer. Valparaíso: Ediciones Universitarias de Valparaíso.

Richards, A. M., y Krauter, E. E. (1999). Cue competition in prospective memory. Psychological Reports, 85(3), 1011-1024.

Sanders, T. (1997). Semantic and pragmatic sources of coherence: On the categorization of coherence relations in context. Discourse Processes, 24, 119-147.

Sanders, T. y Spooren, W. (2001). Text representation as an interface between language and its users. En T. Sanders, J. Schilperoord y W. Spooren (Eds.), Text representation: psycholinguistic aspects (pp.1-26). Amsterdam: John Benjamins.

Sanders, T. y Gernsbacher, M. A. (2004). Accessibility in text and discourse processing. Mahwah, NJ: Erlbaum.

Schwarz-Friesel, M. (2007). Indirect anaphora in text: A cognitive account. En M. Schwarz-Friesel, M. Consten, y M. Knees. Anaphors in Text: Cognitive, formal and applied approaches to anaphoric reference (pp. 3-20). Amsterdam: John Benjamins. 
Schwarz-Friesel, M., Consten, M., y Knees, M. H. (2007). Anaphors in Text: Cognitive, formal and applied approaches to anaphoric reference. Amsterdam: John Benjamins.

Shettleworth, S. J., y Sutton, J. E. (2005). Multiple systems for spatial learning: dead reckoning and beacon homing in rats. Journal of Experimental Psychology: Animal Behavior Processes, 31(2), 125.

Stevenson, R. y Vitkovitch, M. (1986). The comprehension of anaphoric relations. Language and Speech, 29, 335-60.

Swales, J. (1990). Genre analysis. English in academic and research settings. Cambridge: Cambridge University Press.

Swales, J. (2004). Research Genres: Exploration and applications. Cambridge: Cambridge University Press.

Swanson, W. (2003) Modes of Co-reference as an Indicator of Genre. Bérgamo: University of Bergamo.

Vadillo, M. A., Castro, L., Matute, H., y Wasserman, E. A. (2008). Backward blocking: The role of within-compound associations and interference between cues trained apart. The Quarterly Journal of Experimental Psychology, 61(2), 185-193.

Van den Broek, P. (1994). Comprehension and memory of narrative text: Inference and coherence. En M. Gernsbacher (Ed.) Handbook of Psycholinguistics (pp. 539-588). San Diego, California: Academic Press.

Vandorpe, S., De Houwer, J., y Beckers, T. (2007). The role of memory for compounds in cue competition. Learning and Motivation, 38(3), 195-207.

Van Gompel, R. y Liversedge, S. (2003). The influence of morphological information cataphoric pronoun assignment. Journal of Experimental Psychology: Learning, Memory and Cognition, 29(1), 128-139.

Yekovich, F. R., Walker, C. H., y Blackman, H. S. (1979). The role of presupposed and focal information in integrating sentences. Journal of Verbal Learning and Verbal Behavior, 18, 535-548. 
RLA. Revista de Lingüística Teórica y Aplicada, 53 (2), II Sem. 2015

\section{ANEXO 1: EJEMPLO DE RUTINA EXPERIMENTAL}

\section{Diapositiva 1}

\section{Ejemplo}

Pregunta orientadora:

¿A qué hace referencia "ellas" en el siguiente texto?

Presione el botón continuar

Diapositiva 2

\section{Ejemplo}

Texto:

Según Jodelet, en las representaciones sociales interviene lo social de diversas maneras: por el contexto en el cual se sitúan personas y grupos; por la comunicación que situan personas y grupos; por la comunicación que les brinda su bagaje cultural; por los códigos, valores e ideologías ligados a posiciones o pertenencias especificas.

Presione el botón continuar

Diapositiva 3

\section{Ejemplo}

Alternativas:

"Ellas" hace referencia a:

1) Representaciones sociales

2) Personas

3) Posiciones o pertenencias especificas 